\section{SMART PHONE APP TO PROMOTE CORRECT CAR SEAT USE: RESULTS FROM A RANDOMISED CONTROLLED TRIAL}

${ }^{1}$ Eileen M McDonald, 'Wendy C Shields, ${ }^{1}$ Elise Omaki, ${ }^{1}$ Nick Rizzutti, ${ }^{1}$ Patricia Mahoney, ${ }^{2}$ Beverly Miller, ${ }^{2} \mathrm{~S}$ Hope Mullins, ${ }^{2}$ Mary Aitken, ${ }^{1}$ Andrea C Gielen. ${ }^{1}$ Johns Hopkins Centre for Injury Research and Policy, Johns Hopkins Bloomberg School of Public Health, Baltimore, Maryland; ' Injury Prevention Centre, Arkansas Children's Hospital, Little Rock, Arkansas, USA

\subsection{6/injuryprev-2016-042156.120}

Background Child safety seats are known to reduce the risk of death from motor vehicle crashes by between 50\%-80\%. CSS use among younger children is common, use for older children is low, and CSS misuse is widespread. We developed a smart phone app to promote correct CSS use among children younger than 8 years old and tested it with parents of children who presented in one urban and one rural paediatric emergency department in 2 US states.

Methods Research staff helped enrolled parents download the Safety in Seconds app to their smart phone. Parents completed a series of assessment items about their CSS knowledge and beliefs. Intervention group parents reported in more detail about their CSS beliefs and practices with all age-eligible children while control parents answered a similar number of items about another safety topic. Both groups received an electronic report from the app in real time that offered recommendations for improving their health behaviour of interest. All parents received periodic text messages and completed follow up reports through the app at 3 and 6 months. Study enrollment is expected to end in January 2016 and follow ups by July 2016.

Results To date, 761 parents completed baseline surveys $(32 \%$ rural; $68 \%$ urban). Study parents, on average, are black (65.6\%), employed (65.8\%), with more than a high school education (60.0\%), with no differences between study groups. Index child is 5.2 years old. At 6 month follow up, more intervention group parents had gotten their child's safety seat inspected by a car seat technician (34\% vs $24 \%$ ) and properly identified that children should ride in the back seat of the car until at least age $13(35 \%$ vs $27 \%$ ). Additional CSS knowledge, beliefs and practices will be explored when the final data set is ready.

Conclusions Child passenger safety remains an important public health problem. Programs are needed to correct widespread misuse and to promote CSS use among older children.

\section{USING PEER COMMUNICATED BEHAVIOURAL NORMS ABOUT SAFETY TO REDUCE INJURY-RISK BEHAVIOURS BY CHILDREN}

Barbara Morrongiello, Mackenzie Seasons, Ekaterina Pogrebtsova, Julia Stewart, Jayme Feliz. University of Guelph, Guelph Ontario, CANADA

10.1136/injuryprev-2016-042156.121

Background Previous research has shown that children engage in greater physical risk taking when in an elevated positive mood state. The current study examined whether exposure to a peercommunicated behavioural norm about safety could counteract this effect.

Methods Community recruitment resulted in a sample of 120 children ( 7 to 10 years), including 60 boys $(M$ age $=8.13$ yrs; $S D=0.93$ yrs $)$ and 60 girls $(M$ age $=8.02$ yrs; $S D=0.91$ years $)$. Children's intentions to engage in risk taking (based on identifying from photos which risky playground behaviours they would do if they had to make a videotape later that day) and actual risk behaviours (based on how they behaved when running through an obstacle course that contained hazards) were measured while in a neutral and positive mood state, with positive mood induced experimentally via false positive feedback during the playing of a novel videogame (emotion ratings throughout the session validated the positive mood induction worked; there was a significant increase in positive mood, as expected, $t(119)=15.12$, $p<.001)$. Before completing the risk taking tasks when in a positive mood state, children were exposed to either a peer-communicated behavioural norm about safety or a non-norm communication; this exposure occurred by the child overhearing two children supposedly talking next door (this was actually an audiotaped recording).

Results Exposure to the non-norm communication had no effect on risk taking: children showed an increase in risk taking and intentions when in a positive aroused mood state compared to a neutral mood state $(M$ change $=+0.65$ standardised RT score), $F$ $(1,59)=71.31, p<.001, \eta_{\mathrm{p}}{ }^{2}=0.55$. In contrast, exposure to the peer-communicated behavioural norm about safety was effective to counteract this effect: children actually showed a significant decrease in risk taking and intentions when in a positive compared to neutral mood state $(M$ change $=-0.47$ standardised RT score), $F(1,115)=84.77, p<.001$, effect size $\eta_{\mathrm{p}}{ }^{2}=0.42$. Both risk taking measures yielded the same effects.

Conclusion Manipulating children's exposure to peer-communicated behavioural norms can be an effective strategy for reducing injury-risk behaviours.

\section{HOW DO CHILDREN LEARN TO CROSS THE STREET? THE PROCESS OF PEDESTRIAN SAFETY TRAINING}

${ }^{1}$ David C Schwebel, ${ }^{2}$ Jiabin Shen, ${ }^{3}$ Leslie A McClure. ${ }^{1}$ University of Alabama at Birmingham USA; ${ }^{2}$ Nationwide Children's Hospital, Columbus, OH, USA; ${ }^{3}$ Drexel University, Philadelphia, $P A$, USA

\subsection{6/injuryprev-2016-042156.122}

Background Pedestrian injuries are a leading cause of child death, and they may be reduced by training children to cross streets more safely. Training is most effective when children receive repeated practice at the complex cognitive-perceptual task of judging moving traffic and selecting safe crossing gaps, but limited data inform how much practice is required for children to reach adult levels of functioning. Using existing data, we examined how children's pedestrian skills changed over the course of six pedestrian safety training sessions.

Methods As part of a randomised controlled trial on pedestrian safety training, 59 children ages $7-8$ crossed streets within a semiimmersive virtual pedestrian environment 270 times over a 3week period (6 sessions of 45 crossings each). Feedback was provided after each crossing, and traffic speed and density advanced as children's skills improved. Baseline and post-intervention pedestrian behaviours were assessed in the virtual environment.

Results Over the course of training, children entered traffic gaps more quickly and chose tighter gaps to cross within; their crossing efficiency appeared to increase. Post-intervention performance was superior to baseline and by the end of training, some aspects of children's pedestrian behaviour were comparable to adult behaviour (e.g., attention to traffic; start delay - the time between safe traffic gaps appearing and children's entry into those gaps). However, other aspects were not (e.g., collisions with oncoming vehicles). 